UDK 821.131.1-31.09Queneau R.

\title{
LES ONIRIQUES FLEURS BLEUES DE RAYMOND QUENEAU
}

\author{
Primož Vitez
}

\begin{abstract}
Résumé
Dans Les fleurs bleues (1965) de Raymond Queneau, le narrateur fait couler deux histoires parallèles qui se terminent dans l'unité du présent énonciatif. L'effet contrastant de ce double récit, habité d'un côté par le Duc d'Auge et de l'autre par un certain Cidrolin, deux facettes d'un seul personnage, se fonde sur un principe onirique, déclenché au moment quand le Duc d'Auge s'endort; dans son rêve se déploie l'histoire de Cidrolin qui finit par s'endormir à son tour - et voilà le lecteur replongé dans les aventures du Duc. Ce que l'auteur développe par ses astuces narratives, c'est la pluralité du réel, ce sont les parallélismes invisibles, c'est la promesse d'une perspective existentielle, c'est la fécondité de l'ironie. À travers la variation de ce qui apparamment est ordinaire, Queneau démontre une dimension extraordinaire de l'invention littéraire.
\end{abstract} variation

Mots-clés : Raymond Queneau, Les fleurs bleues, récit onirique, fiction, réalité littéraire,

Figurez-vous que tout à l'heure, en dormant, j'ai fait un rêve étrange. J'étais un papillon voltigeant, ivre de lumière et du parfum des fleurs. Et maintenant, je ne sais plus si je suis Tchouang-tseu ayant rêvé qu'il était un papillon ou un papillon qui rêve qu'il est Tchouang-tseu!

(Le rêve du papillon, Contes et histoires zen)

La dramaturgie ${ }^{72}$ du meilleur roman (1965) de Queneau, dans ses éléments, est simple : de chapitre en chapitre, ils sont au nombre de vingt-et-un, on poursuit l'écoulement de deux histoires parallèles qui finissent par confluer en une seule. L'effet contrastant de ce double récit, habité d'un côté par le Duc d'Auge et de l'autre par un certain Cidrolin, se fonde sur un principe onirique, annoncé par l'exergue, tiré du Théétète

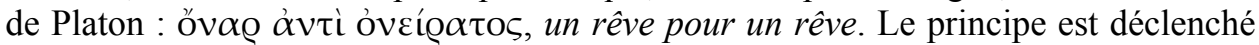
au moment quand le Duc d'Auge, pour la première fois du roman, s'endort; dans son

${ }^{72}$ On dira bien dramaturgie, car Queneau, ici encore, est filmique. 
rêve se déploie l'histoire de Cidrolin qui finit par s'endormir à son tour - et nous voilà replongés dans les aventures du Duc. Le hameçon narratif est lancé et peu à peu on perd la trace de savoir qui dort et qui fait l'apparition onirique; qui rêve et qui est rêvé; qui est le créateur du rêve et qui en est la créature; qui est l'auteur ensommeillé et qui est le personnage de son imagination.

Ce double récit réalise une dialectique du monde, s'élaborant dans un dialogue entre le présent universel et les époques historiques particulières; entre les deux côtés d'une même histoire personnelle, celle du héros principal, appelé tour à tour Duc d'Auge ou Cidrolin. C'est une construction ingénieuse d'un personnage en tant qu' amalgame de son histoire actuelle et de son fond historique et traditionnel. La structure de l'ouvrage rappelle le potentiel associatif et unificateur des deux grandes séries des Écritures chrétiennes où l'Ancien Testament n'est pas seulement la précursion de ce qu'apportera le Nouveau, mais son vrai pendant historique. Le principe que l'exégèse biblique définit en termes de Concordia veteris et novi Testamenti se trouve reformulé dans le roman de Queneau comme une élaboration raffinée d'une personne fêlée et de son histoire fourvoyée, comme une certaine Concordia historiae et personnae praesentis. Que le Duc d'Auge et Cidrolin se rêvent, est une allusion singulière à l'onirisme en tant qu'écran audiovisuel de l'inconscient, qui détermine - plus que toutes les actions conscientes - la condition humaine : que l'homme est une ambiguité, un paradoxe sur ses deux jambes qui ne saurait comprendre qui il est, d'où il vient, comment il s'en sortira et pourquoi il fait ce qu'il lui arrive de faire. Plus il semble s'approprier le sentiment de comprendre la vie et de pouvoir faire passer sa connaissance ailleurs, vers les autres, plus il se trouve délaissé par la certitude, moins il ressent de stabilité dans son savoir, plus il devient suspicieux et rongé par le doute.

À quoi bon ici cette incursion dialectique, cette vieille histoire de la nature humaine, inévitablement double, rappel de la célèbre fresque baudelairienne de la plaie qui en même temps est un couteau, du bourreau et sa victime, réunis dans un seul être ? Cidrolin habite sur l'eau, sur une péniche accostée au rivage de la Seine - comme on en trouve encore aujourd'hui à Saint-Ouen - et protégée par une haie en bois où chaque nuit, depuis des années, un anonyme vient écrire des graffiti insultants à son sujet. Son seule constante activité quotidienne, à part la consommation de l'essence de fenouil, c'est de recouvrir de couleur fraîche ces insultes nocturnes. Dans le dénouement dramatique du roman on apprend que l'auteur de ces injures peintes n'est autre que Cidrolin lui-même, qu'il vient s'insulter sur son propre wall, pour se sentir ensuite obligé de le repeindre et de diminuer la honte. Il ne reste plus rien de cet anonymat commode qui lui permettait pourtant de musculariser du jour au jour sa douleur. Il est particulièrement gênant pour lui de voir que c'est son pendant historique, son alter ego, le Duc d'Auge qui dans une action éclatante révèle son mystère et démasque l'auteur non moins honteux que ses inscriptions. Il n'y a pas lieu ici de s'appuyer excessivement, en interprétant cette métaphore bizarre, aux notions psychanalytiques, telles que scission, manque, fêlure et ainsi de suite - mais il n'en reste pas moins vrai que c'est plus ou moins de cela qu'il s'agit. Il faut constater que le pinceau de quelqu'un qui la nuit s'insulte graphiquement pour s'excuser le jour, dessine un tableau tant soit peu romantique de l'être créatif réunissant l'essentiel de toutes les oppositions, voire contradictions morales. Comme sa vie se présente comme un dilemme, il ne se permettra pas de se conformer aux parti-pris trop 
prévisibles, il ne défend ni l'un ni l'autre côté de sa controverse intime, mais essaiera plutôt d'orienter sa foi, comme la raison d'ailleurs, vers le dilemme tout court. L'image de l'artiste qui, aux moments critiques du processus créatif, est capable de s'adresser du mépris, de s'anéantir en se dévalorisant douloureusement, de se cracher au visage et de s'inonder compulsivement au pastis - tout cela pour s'affermir à long terme, pour densifier la longue attente de l'occasion transcendante qui lui permette, tel oiseau ressuscité des cendres, de remonter sa frustration et de croire moins nerveusement en l'avènement du jour de son ascension vers l'éternité.

L'ironie clairvoyante, l'intelligence que l'auteur établit vis-à-vis de son héros para(llé)lysé, et, indirectement bien sûr, vis-à-vis de soi-même, c'est ce qui enveloppe cette histoire onirique dans tout son éclat narratif. Car le rêve permet les combinaisons les plus asticieuses de tout ce qui, pendant les années de sa vie, s'est accumulé dans la mémoire accessible et encryptée du rêveur; voilà pourquoi le récit, à un moment donné, se trouve agrémenté par une idée singulière, d'après laquelle qu'il y a de l'indécence à vouloir raconter ses rêves à autrui. Dans le rêve il n'y a pas d'incertitude, tout ce qui s'y passe est dépourvu de doute, mais non d'ambiguité : c'est pourquoi, depuis toujours, on ne voit dans le rêve que des images allusives; des images qui se racontent, certes, mais qui en même temps racontent autre chose. Les rêves s'ouvrent donc immédiatement sur l'interprétation, démystifiant en détail les couches psychologiques et caractérielles du rêveur. Le risque qu'on court de dire ses rêves, c'est le risque que court l'écrivain en publiant ses textes. Mais si le sommeil, apportant le rêve, dévoile la condition émotive du rêveur et ses obsessions, il existe un autre état qui, lui, intensifie les processus intellectuels. C'est la phase intermittente entre l'éveil et le sommeil, le temps d'assoupissement, quand les pensées d'une conscience intéressée restent en vigueur, mais se trouvent graduellement envahies par la haute-marée d'une paix rassurante qu'apporte l'emprise de l'inconscient. Dans cette confluence d'avant-sommeil, dans ces minutes bénies quand le fleuve de la conscience s'unit à la mer des mémoires oubliées, on dresse des plans, on voit remonter des structures, on jette des projections; les schémas s'assemblent, les chiffres s'agitent. La lucidité de cet état révélateur conserve au roseau pensant le potentiel de sa connaissance et devient la source d'une cohérence originale, réaffirmant la prémonition des structures sur lesquelles se fonde la conviction de toute oeuvre d'art bien énoncée.

Il semble que dans la conception des Fleurs bleues, ce sont encore les chiffres qui se sont le plus agités, et parmi eux le sept qui s'avère particulièrement prodigieux. Queneau se servait abondamment du chiffre sept dans ses ouvrages de jeunesse; c'est d'ailleurs le nombre de lettres que comportent son nom et son prénom. Le parallèle historique de l'histoire de Cidrolin, le récit onirique du Duc d'Auge, s'étend sur sept siècles de l'histoire française, de 1264 jusqu'à l'an 1964, temps actuel de la création du roman. Ces sept cents ans s'écoulent fictivement en vingt-et-un chapitres (ce qui est divisible par sept), et dans la structure narrative de cette double histoire toute la longue époque est partagée entre quatre intervalles de 175 ans. Au début de la septième décennie de sa vie (il a 61 ans en 1964), Queneau fait le geste de se rappeler sa jeunesse créatrice : à chaque époque historique, du temps du roi Saint-Louis (1264) à l'actuel (1964), en passant par la Renaissance (1439), par le classicisme naissant (1614) et par la Révolution (1789), il s'écoule sept fois vingt-cinq ans ce qui chez l'homme pourrait 
ressembler à un âge qu'on appelle jeunesse. Ces astuces arythmétiques finissent donc par se transformer en une réflexion du passage et de la finitude, en un examen de la temporalité et - pourquoi non - de la durée. Il s'agit pour Queneau de se demander, comment coule le temps dans une histoire personnelle, dans l'histoire d'un héros littéraire, comment coule le temps du monde, le temps de «l'histoire universelle en général et de l'histoire générale en particulier», mais surtout - quel est le cours du temps dans un récit littéraire : comment l'auteur peut saisir le temps pour lui donner une forme et comment le lecteur peut le vivre sans qu'il soit saisi au cou. Queneau formule le temps en formulant son écriture : il n'attribue pas aux choses fatales une fatalité a priori, il prend son propre temps à les énoncer dans des gestes consécutifs d'esprit, permettant au lecteur de sourire et de relâcher de temps en temps ses muscles faciales en un rire libérateur. Le rire intervient surtout quand l'auteur réfléchit directement, à travers l'écriture, sa propre méthode narrative. On rit quand l'écriture, pour ainsi dire, se joue de soi-même pour devenir élégamment métagraphique. Parmi les passages qui exercent à merveille la capture du temps, en voilà un qui nous semble particulièrement réussi :

L'abbé saisit la corde et emboîte le pas au duc qui tient la lanterne à la main. Ila avancent en silence.

Dans le silence obscur, ils avancent.

Dans l'obscurité silencieuse, ils continuent d'avancer.

Sans cadence, ils avancent, la corde se balance et la lanterne aussi, c'est toujours le silence.

Ce n'est pas tout à fait le silence, car il y a le bruit des pas, ce n'est pas tout à fait l'obscurité, car il y a cette petite lumière au bout du bras du conducteur.

Ils avancent en silence.

Soudain :

- Monsieur le duc...

- Ne craignez rien, l'abbé, vous voyez bien que je suis toujours là.

C'est un geste réflexif de l'auteur s'aventurant dans une fusion de son énonciation avec l'énoncé, reproduisant en même temps le cheminement du temps et le dynamisme des événements écrits, unissant la description au décrit : pour Queneau, ici, avancer dans le silence, c'est exécuter cette démarche obscure et silencieuse qui est celle d'écrire. À cette brillante analyse simultanée du temps réel, fictif, narratif et perceptif se joint la finesse d'une harmonie phonique (avancent-silence-balance), un mouvement poétique dans l'esprit de l'écart comique et intelligent qu'effectue l'auteur par rapport aux prosations stéréotypées. Mais où qu'on se décide de mettre l'oeil, quelle que soit l'astuce observée de l'écrivain : ses imaginations orthographiques, ses néologismes et inventions néo-babéliennes, ses structures polyphoniques, produisant des effets tropiques (on est presque tenté de dire poésiques) qui augmentent la tension hospitalière de la lecture il y a au-dessous de toutes ces perles narratives la langue. Et pas n'importe laquelle : c'est la poésie exceptionnelle de Queneau, inimitable, foncièrement originale. C'est le français quenélien, un idiolecte littéraire inépuisable, monté sur le fond de toutes les pratiques langagières vécues et imaginées. La surface fascinante du texte recèle la vraie fascination de l'auteur : le goût millénaire de la langue française, celui de la variation, toujours et partout, dans le scintillement de la parole, dans les pièces canonisées et in- 
connues de l'impressionnante histoire de la littérature française. De Rabelais aux maîtres du théâtre classique, en passant par les fous littéraires dont Queneau s'établissait une spécialisation. Ils ont tous en commun d'avoir essayé de (se) comprendre. Ils se sont tous donné cette peine, il se sont tous affligé cet effort prestigieux, ils ont tous entamé ce labeur presque surhumain - ils ont pensé.

Le plaisir que ressent Queneau pour la variation constitue le noyau de son écriture et de son engagement artistique. La variation se présente comme un procédé de composition, ${ }^{73}$ dessinant le véritable fil conducteur de sa création originale. Ce penchant variationnel est à la source d'une autre joie quenélienne, celle de l'érudition débridée, mêlée à la construction des labyrinthes de l'intertextualité où seuls les lecteurs bien avisés sont invités à errer. Quand il était question tout à l'heure de parallélismes de la structure narrative et de la dialectique des éléments littéraires, il s'agissait en effet de la variation d'une même chose : Cidrolin et le Duc d'Auge ne sont que variation du même héros, deux figures d'un seul personnage. Le principe variationnel s'imbrique naturellement dans les fondements du roman : les lieux d'embrayage narratif, de passage d'événement en événement, d'histoire en histoire, et puis d'Histoire au Présent, il y a incessamment retour au même - le personnage, à un moment crucial, doit s'endormir pour que son alter ego puisse revivre. Disons au hasard que Cidrolin et son pendant historique s'assoupissent dans le roman vingt-huit ou trente-cinq fois, pour ne pas abandonner la logique structurelle de la multiplication par sept. L'auteur s'est donc délégué une tâche formelle, celle de varier sensiblement la formulation d'un même événement banal. Ce procédé variationnel, on le sait, a été développé avec beaucoup d'esprit dans les Exercices de style - et Queneau ne semble pas y avoir renoncé depuis. L'action fondamentale de la variation, c'est d'ouvrir la potentialité formelle, de s'ouvrir sur l'invention des formes et c'est ce qui représente le motif intemporel de l'art : chercher et finir par trouver le moyen de renouveler, par le regard écrit, les choses qui existent depuis toujours. La vérité abstraite, objectif tacite de toute aventure artistique, ne se confond pas avec la chose elle-même, elle est le chemin véridique, plus ou moins convaincant et reconnu, qui mène vers cette chose, chemin qu'il faut faire en passant par la raison, mais aussi, comme on dit parfois, par le coeur. L'histoire de l'art, c'est l'histoire des formes artistiques.

Le titre du roman, quelque peu intraduisible vers d'autres langues, Les fleurs bleues, est un syntagme conventionnel, mais plutôt rare en français. C'est une expression métaphorique pour un animé, quelqu'un de trop sensible, excessivement sentimental, presque pleurnichard et certainement crédule qui (parfois sous l'influence de l'alcool) s'enthousiasme jusqu'à l'euphorie pour les choses auxquelles il n'oserait pas trop penser en état de sobriété psychologique et physiologique. Le choix du titre est sans motif prévisible ou confirmable par la cohérence de surface textuelle : il n'y apparaît que deux fois, au début et tout à la fin, dans la dernière phrase. On a encore affaire au goût que Queneau éprouve à faire allusion aux fleurs de la littérature française qui ont su inspirer sa création. Du côté sémantique, il y a, certes, la ressemblance avec Les fleurs du mal (1857), célèbre recueil de Baudelaire; en revanche, du point de vue de la pragmatique du

${ }^{73}$ D'une part on comprendra la variation comme un retour incessant à «toujours la même chose», de l'autre, c'est la construction de rapports entre les divers éléments d'une oeuvre d'art, un trait compositif contribuant à l'établissement des dimensions esthétiques du texte. 
texte, ce titre fonctionne comme un geste ludique et arbitraire, rappelant La Cantatrice chauve (1950) de Ionesco, l'antidrame du langage perdu, où le titre ne surgit qu'une seule fois, sans lien directement cohésif à l'action, dans une réplique du Pompier. En cherchant les relations sémantiques du titre avec l'univers romanesque de Queneau, on n'aura peut-être pas trop tort d'insister sur la qualification explicative «parfois sous l'influence de l'alcool». Il est vrai qu'une certaine boisson alcoolique sert de carburant principal au personnage flottant sur l'Arche - et qu'on reproche un peu souvent à Cidrolin qu'il boit trop - et qu'il dort mieux s'il a bu «un tantinet soit peu». Mais il n'en est pas moins vrai que, plus généralement, dans la caractérisation de la fleur bleue l'alcool est l'un des moyens principaux permettant la fugue, l'évasion, la fuite, l'absentéisme, le «foutre le camp» devant la réalité qui, elle, implique la présence souvent inopportune de soi à tout instant. Pour comprendre cette réalité, il ne suffit pas de dire qu'elle n'est pas toujours agréable ou qu'elle risque parfois d'être opprimante; il faut surtout dire que cette réalité ne peut nullement être la seule réalité dans la vie d'un individu. En cherchant l'abri devant cette réalité oppressive, une fleur bleue, effectivement, peut s'exiler impunément dans le rêve que le poète sublime, Gérard de Nerval, qualifie d'une "seconde vie». Dans le rêve, il est permis au rêveur de s'enthousiasmer sans freins pour les variations supra-intellectuelles du bien et du mal, du beau et du laid, du vrai et du soi-disant faux. Il est permis de se poser dans les sphères oniriques du second monde des questions indécentes, telle la question sur l'existence des préadamites, les gens qui existeraient avant Adam et dont on croit, avec pas mal d'humour, retrouver l'image dans les grottes de Montignac, de Lascaux et d'Altamire. En d'autre termes : quel profit pourrait-on tirer de la réconciliation éventuelle de se rapport douteux entre la théologie et la théorie ? Le transfuge vers l'euphorie, dans son imagination onirique, ne s'arrête pas là. Il s'offre le luxe de poser une autre question, non moins pieuse et bien plus éruptive que la première : ne serait-il pas franchement plus profitable pour tous si les plus grands savants (Galileo Galilei) de l'histoire humaine se contentaient tout simplement d'être alchimistes auréogènes (Timoleo Timolei), indépendamment du résultat de l'entreprise scientifique? La promesse d'une telle liberté, arrivée au bord d'un sombre précipice sans fond, ne laisse d'ouverte que la question de technique : comment s'endormir ? Comme s'endormir pour que la pensée soit transparente?

Que les sept cents ans de rêve historique dans Les fleurs bleues se joignent au moment actuel (1964) de l'histoire narrée, c'est tout de même une conclusion optimiste par rapport à l'ordinaire réaliste, voire pessimiste des perspectives existentielles. Il semble que la sagesse de l'auteur s'apprête à lever le doigt sur la coexistence de diverses réalités (soient-elles parallèles), d'univers pluriels dans ce que l'on perçoit habituellement - et par erreur - comme la seule réalité, comme le seul (et du coup le meilleur) monde possible, comme la seule combinaison concevable des coordonnées spatio-temporelles. Si on s'abandonne à ne regarder notre présent que sous ce pauvre angle, si c'est par là que l'on se construit notre «point zéro» en tant que catégorie temporelle exclusive (qui, disons-le en passant, n'existe pas), cela signifie qu'on se défend d'être entiers. On renonce par là à la possibilité d'une auto-révélation dans la gloire de notre histoire intime et culturelle. On se prive de tout ce que l'on pourrait nommer salut quotidien de l'imagination, ainsi que de toute chance de consommer jusqu'au fond la puissance de notre langage. Dans son roman, Queneau offre au lecteur cette possibilité 
d'identification oubliée : se construire un panorama des sphéres invisibles que l'on a pris l'habitude d'ignorer.

\author{
Université de Ljubljana, Slovénie
}

\title{
BIBLIOGRAPHIE
}

Barthes, Roland. Le plaisir du texte. Paris : Seuil, 1982.

Ionesco, Eugéne. Notes et contre-notes. Paris : Gallimard, 1966.

Jakobson, Roman. Six leçons sur le son et le sens. Paris : Minuit, 1976.

Jarry, Alfred. Gestes et opinions du docteur Faustroll, pataphysicien. Paris : Fasquelle, 1911.

Jouet, Jacques. Raymond Queneau. Paris, La manufacture, 1989.

Longre, Jean-Pierre. Raymond Queneau en scènes. Limoges : Presses Universitaires de Limoges, 2005.

Pouilloux, Jean-Yves. Les fleurs bleues de Raymond Queneau. Paris : Gallimard, 1991. 